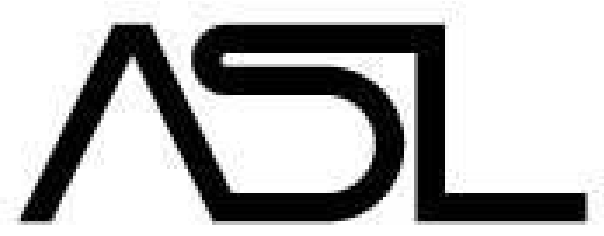

Uniform Upper Bounds on Ideals of Turing Degrees

Author(s): Harold T. Hodes

Source: The Journal of Symbolic Logic, Sep., 1978, Vol. 43, No. 3 (Sep., 1978), pp. 601612

Published by: Association for Symbolic Logic

Stable URL: https://www.jstor.org/stable/2273535

JSTOR is a not-for-profit service that helps scholars, researchers, and students discover, use, and build upon a wide range of content in a trusted digital archive. We use information technology and tools to increase productivity and facilitate new forms of scholarship. For more information about JSTOR, please contact support@jstor.org.

Your use of the JSTOR archive indicates your acceptance of the Terms \& Conditions of Use, available at https://about.jstor.org/terms

Association for Symbolic Logic is collaborating with JSTOR to digitize, preserve and extend access to The Journal of Symbolic Logic 


\section{UNIFORM UPPER BOUNDS ON IDEALS OF TURING DEGREES ${ }^{1}$}

\section{HAROLD T. HODES}

§1. Preliminaries. Given $I$, a reasonable countable set of Turing degrees, can we find some sort of canonical strict upper bound on $I$ ? If $I=\{\underline{a} \mid \underline{a} \leq \underline{b}\}$, the upper bound on $I$ which springs to mind is $\underline{b}^{\prime}$. But what if $I$ is closed under jump? This question arises naturally out of the question which motivates a large part of hierarchy theory: Is there a canonical increasing function from a countable ordinal, preferably a large one, into $D$, the set of Turing degrees? If $d$ is to be such a function, it is natural to require that $d(\alpha+1)=d(\alpha)^{\prime}$; but how should $d(\lambda)$ depend on $d\lceil\lambda$, where $\lambda$ is a limit ordinal?

For any $I \subseteq D$, let $M_{I}=\bigcup I$. Towards making the above questions precise, we introduce ideals of Turing degrees.

DEFINITION 1. $I \subseteq D$ is an ideal iff $I$ is closed under jump and join, and $I$ is downward-closed, i.e., if $\underline{a} \leq \underline{b} \& \underline{b} \in I$ then $\underline{a} \in I$.

The following definition reflects the hierarchy-theoretic motivation for this paper.

Definition 2. For $I \subseteq D$ and $A \subseteq \omega, I$ is an $A$-hierarchy ideal iff for some countable ordinal $\alpha, M_{I}=L_{\alpha}[A] \cap \omega^{\omega}$.

All hierarchy ideals are ideals, but not conversely.

Early in the game Spector knocked out the best sort of canonicity for upper bounds on ideals, proving that no set of degrees closed under jump has a least upper bound. In search of some sort of canonicity, uniform upper bounds were introduced in [1] and [4]; nice uniform upper bounds were introduced in [2]. In this chapter, we characterize nice uniform upper bounds on countable ideals; we point out a relationship between such upper bounds and degree hierarchies supported by systems of notation; we answer several natural questions about leastness of such upper bounds.

Notation and definitions. " $f$ ", etc. range over $\omega^{\omega}$; " $A$ ", etc. range over $P(\omega)$; " $\underline{a}$ ", etc. range over $D$.

" $\leq$ " (" $<")$ ambiguously represents Turing (proper Turing) reducibility on $D, P(\omega)$ and $\omega^{\omega}$. Fix a recursive pairing function $\lambda x y .\langle x, y\rangle$ from $\omega \times \omega$ onto $\omega$, with left (right) inverses $\lambda x . l(x)(\lambda x . r(x))$.

$\operatorname{Graph}(f)=\{\langle x, f(x)\rangle \mid x \in \omega\}$. As usual, " $\{n\}$ " abbreviates " $\{n\}^{\text {graph(f) }) "}$. $(f)_{i}(x)=f(\langle i, x\rangle) . f \oplus g=h$ such that $(h)_{0}=f,(h)_{1}=g,(h)_{i+2}=\lambda x .0$ for all $i$. Str is the set of strings, finite sequences of 0 's and 1's coded as natural numbers.

Received May 4, 1976.

${ }^{1}$ Theorem $1,(1) \Leftrightarrow(2)$, and Theorem $2,\left(1^{*}\right) \Leftrightarrow\left(2^{*}\right)$, and possibly several other results reported here were obtained independently by Saul Kripke. Thanks to Andreas Blass, George Boolos, Carl Jockusch, Hilary Putnam and Steve Simpson for suggestions and encouragement. 
" $\sigma$ ", " $\tau$ ", etc. range over Str. $\sigma \subseteq \tau$ iff $\sigma$ is an initial segment of $\tau$; $\operatorname{lh}(\sigma)$ is the length of $\sigma ; \tau=\sigma^{n} n$ iff $\operatorname{lh}(\tau)=\operatorname{lh}(\sigma)+1, \sigma \subseteq \tau$, and $(\tau)_{\operatorname{lh}(\tau)-1}=n$. Let $\sigma \subseteq A$ iff $(\forall i<\operatorname{lh}(\sigma))\left((\sigma)_{i}=1\right.$ iff $\left.i \in A\right)$. Let $\hat{\sigma}=\left\{i \mid i<\operatorname{lh}(\sigma) \&(\sigma)_{i}=1\right\}$. We shall need the notion of a computation in a partial object. $\{n\}^{*^{\sigma}}(x)$ is $\{n\}^{\hat{\sigma}}(x)$, provided that the latter computation asks the oracle no questions of the form " $i \in \hat{\sigma}$ ?" for $i \geq \operatorname{lh}(\sigma)$; it diverges otherwise. Similarly, $\{n\}^{*^{A \oplus \sigma}}(x)$ is $\{n\}^{\mathbf{A} \oplus \hat{\sigma}}(x)$, provided the latter computation asks the oracle no questions of the form " $i \in A \oplus \hat{\sigma}$ ?" for $i$ such that $r(i) \geq \operatorname{lh}(\sigma)$; it diverges otherwise.

Let $J_{\underline{a}}=\{\underline{b} \mid \underline{b} \leq \underline{a}\}$. The following is well known: $I$ is a countable ideal iff $I$ is closed under jump and for some $\underline{a}$ and $\underline{b}, I=J_{\underline{a}} \cap J_{\underline{b}}$. If $I=J_{\underline{a}} \cap J_{\underline{b}},(\underline{a}, \underline{b})$ is $I$-exact, or an exact pair for $I$.

Definition 3. For $X \subseteq \omega^{\omega}, f$ parametrizes $X$ iff $X=\left\{(f)_{i} \mid i \in \omega\right\}$. In this case, $n$ is an $f$-index of $(f)_{n}$.

DEFinition 4. For $J \subseteq D, \underline{a}$ is a uniform upper bound (hereafter u.u.b.) on $J$ iff $\underline{a}$ is the degree of a parametrization of $M_{J} ; \underline{a}$ is a weak u.u.b. iff $\underline{a}$ is the degree of a parametrization of $M_{J} \cap 2^{\omega}$.

Definition 5. Let $f$ parametrize $X \subseteq \omega^{\omega}$. If $X$ is closed under jump, $g$ is an $f \leq$ jump locator iff for any $n,(f)_{g(n)}=(f)_{n}^{\prime}$. If $X$ is closed under join, $g$ is an $f$-join locator iff for any $n$ and $m,(f)_{g(n, m)}=(f)_{n} \oplus(f)_{m} . f$ nicely parametrizes $X$ iff there are $f$-jump and $f$-join locators recursive in $f$. A nice parametrization of $X$ is nice because: if $\phi$ is an arithmetic formula with $k$ free function variables, $\phi\left((f)_{n_{1}}, \ldots,(f)_{n_{k}}\right)$ is decidable in $f$.

Definition 6. For $J \subseteq D$ and closed under jump and join, $\underline{a}$ is a nice u.u.b. (a nice weak u.u.b.) iff $\underline{a}$ is the degree of some nice parametrization of $M_{J}$ (of $\left.M_{J} \cap 2^{\omega}\right)$.

Observations. (1) $\underline{a}$ is a nice weak u.u.b. on $J$ iff $\underline{a}$ is a nice u.u.b. on $J$.

(2) If $\underline{a}$ is a weak u.u.b. on $J$ then $\underline{a}^{\prime}$ is a u.u.b. on $J$.

(3) If the definition of a nice parametrization were changed by requiring that the jump and join locators be recursive, the resulting definitions of nice u.u.b.s and nice weak u.u.b.s would still coincide with the given definitions.

Proof. Use the $s-m-n$ theorem. This definition of a weak u.u.b. is equivalent to the definition of a u.u.b. provided in [1] and [4]; this definition of a nice weak u.u.b. coincides with the definition of a nice bound provided in [2].

(4) If $\underline{a}$ is a u.u.b. (weak u.u.b.) (nice u.u.b.) on $J$ and $\underline{b} \geq \underline{a}$, then $\underline{b}$ is a u.u.b. (weak u.u.b.) (nice u.u.b.) on $J$.

PRoOF. If $f \in \underline{a}$ parametrizes $M_{J}$ and $B \in \underline{b}$, define $g$ such that $(g)_{2 i+1}=$ $(f)_{i},(g)_{2 i}=\lambda x .0$ if $i \in B$, and $\lambda x .1$ otherwise. Any $f$-jump or $f$-join locator recursive in $f$ can be readjusted to yield a $g$-jump or $g$-join locator recursive in $g$.

\section{§2. Nice uniform upper bounds.}

THEOREM 1. Let I be a countable ideal. The following are equivalent:

(1) $\underline{a}$ is a nice u.u.b. on $I$;

(2) for some $\underline{b}$ and $\underline{c},(\underline{b}, \underline{c})$ is exact for $I$ \& $\underline{a}=(\underline{b} \cup \underline{c})^{(2)}$;

(3) for some $\underline{b}, \underline{b}$ is a u.u.b. on I \& $\underline{b}^{\prime}=\underline{a}$;

(4) for some $\underline{b}, \underline{b}$ is a weak u.u.b. on $I \& \underline{b}^{(2)}=\underline{a}$. 
(2) $\Rightarrow$ (3) Let $(\underline{b}, \underline{c})$ be exact for $I, B \in \underline{b}, C \in \underline{c}$. It suffices to show that $(\underline{b} \cup \underline{c})^{\prime}$ is a u.u.b. on $I$. Let

$$
(g)_{\langle n, m\rangle}(x)= \begin{cases}\{n\}^{B}(x) & \text { if }(\forall y \leqslant x)\left(\{n\}^{B}(y) \text { and }\{m\}^{C}(y)\right. \text { converge } \\ & \left.\&\{n\}^{B}(y)=\{m\}^{C}(y)\right), \\ 0 & \text { otherwise. }\end{cases}
$$

If $n^{B}(x)$ is not total or $\{n\}^{B} \neq\{m\}^{C},(g)_{\langle n, m\rangle}$ is nonzero on a finite number of arguments, and so belongs to $M_{I}$. Thus for any $n$ and $m,(g)_{\langle n, m\rangle} \in M_{I}$. Thus $g$ parametrizes $M_{I}$.

(3) $\Rightarrow$ (1) Let $\underline{b}$ be a u.u.b. on $I ; f \in \underline{b}$ parametrizes $M_{I}$.

Claim. $\left\{\langle m, n\rangle \mid(f)_{m}=(f)_{n}{ }^{\prime}\right\}$ is $\Delta_{2}^{0}$ in $f$. The $\Pi_{2}^{0}$ in $f$ formulation is obvious. $(f)_{m}=(f)_{n}{ }^{\prime}$ iff $(\forall x)(\exists y) Q\left(x, y,(f)_{m},(f)_{n}\right)$ iff $\left(\exists h \in M_{I}\right)(\forall x) Q\left(x, h x,(f)_{m},(f)_{n}\right)$ iff $(\exists y)(\forall x) Q\left(x,(f)_{y}(x),(f)_{m},(f)_{n}\right)$. Thus there is an $f$-jump locator recursive in $f^{\prime}$. Similarly, there is an $f$-join locator recursive in $f^{\prime}$. We can then code $f^{\prime}$ into $f$ and readjust these locators to the result, as in the proof of observation (4), insuring that the resulting locators remain recursive in $f^{\prime}$.

(4) $\Rightarrow$ (3) This is immediate from observation (2).

It shall be convenient to prove that (1) implies each of:

$\left(2^{\prime}\right)$ for some $\underline{b}$ and $\underline{c},(\underline{b}, \underline{c})$ is exact for $I \&(\underline{b} \cup \underline{c})^{(2)} \leq \underline{a}$;

$\left(3^{\prime}\right)$ for some $\underline{b}, \underline{b}$ is a u.u.b. on $I \& \underline{b}^{\prime} \leq \underline{a}$;

(4') for some $\underline{b}, \underline{b}$ is a weak u.u.b. on $I \& \underline{b}^{(2)} \leq \underline{a}$; and then prove that $\left(2^{\prime}\right) \Rightarrow$ $(2),\left(3^{\prime}\right) \Rightarrow(3),\left(4^{\prime}\right) \Rightarrow(4)$.

$(1) \Rightarrow\left(2^{\prime}\right)$ This proof uses forcing with uniformly recursively pointed perfect conditions in an arithmetic setting. All terminology and basic lemmas may be found in [3]. $P \in \omega^{\omega}$ is perfect iff $P$ "Str $\subseteq$ Str \& (for any $\sigma, P\left(\sigma^{n} 0\right)$ and $P\left(\sigma^{n} 1\right)$ are incompatible extensions of $P(\sigma))$. View a perfect $P$ as a binary tree of strings without finite branches, each branch coding a member of $2^{\omega}$. [P] is the set of reals coded by branches of $P . P$ is uniformly recursively pointed iff $(\exists n)$ $(\forall A \in[P])\left(P=\{n\}^{A}\right) .(P, Q)$ is a condition iff $P$ and $Q$ are uniformly recursively pointed perfect functions, members of $M_{I}$, and $P \equiv_{T} Q$. $(P, Q)$ extends $(R, S)$ iff $P \subseteq R \& Q \subseteq S$, where $P \subseteq R$ iff $(\forall \sigma)(\exists \tau) P(\sigma)$ is an initial segment of $R(\tau)$. [P, $Q]=[P] \times[Q]$.

Construct a forcing language from number variables, numerals, predicate constants for primitive recursive predicates on $2^{\omega} \times 2^{\omega} \times \omega$, and generic constants " $\underline{B}$ " and " $\underline{C}$ "; build prenex sentences from " $\exists$ " and " - ", with the usual $\Pi_{n}^{0}$ and $\Sigma_{n}^{0}$ classification. Call a sentence eligible iff it belongs to $\Pi_{0}^{0} \cup \Sigma_{1}^{0} \cup \Pi_{1}^{0} \cup \Sigma_{2}^{0} \cup \Pi_{2}^{0}$. We define $(P, Q) \Vdash \phi$, where $(P, Q)$ is a condition and $\phi$ is an eligible sentence, as follows:

$$
\begin{aligned}
& (P, Q) \Vdash \phi \text { iff }(\forall(B, C) \in[P, Q])(B, C) \vDash \phi, \quad \text { for } \phi \in \Pi_{0}^{0} \cup \Pi_{1}^{0} \cup \Pi_{2}^{0} \text {; } \\
& (P, Q) \Vdash(\exists x) \phi \text { iff }(\exists n)(P, Q) \Vdash \phi \underline{n}, \quad \text { for } \phi \in \Pi_{0}^{0} \cup \Pi_{1}^{0} .
\end{aligned}
$$

By [3, Lemma 3.5], given $(P, Q)$ and eligible $\phi$, some $(R, S)$ extends $(P, Q)$ and decides $\phi$. Call an $\omega$-sequence of conditions generic iff for each $n$, the $n+1$ st extends the $n$th and each eligible sentence is decided by some condition in the 
sequence. If $\left\langle\left(P_{n}, Q_{n}\right)\right\rangle_{n \in \omega}$ is generic, let its limit be the pair $(B, C)$ such that $\{B\}=\bigcap_{n}\left[P_{n}\right],\{C\}=\bigcap_{n}\left[Q_{n}\right]$. By [3, Lemma 3.7], $\{f \mid f \leq B \& f \leq C\} \subseteq M_{I}$. By $\left[3\right.$, Lemma 3.8], the forcing relation is $\Sigma_{3}^{0}$.

Let $f \in \underline{a}$ nicely parametrize $M_{I} . n$ codes a condition iff $(f)_{n}=P \oplus Q$, where $(P, Q)$ is a condition. We construct a generic sequence of conditions with limit $(B, C)$ and a function $h \leq f$ such that

(i) $h(n)$ codes the $n$th condition in the sequence;

(ii) $M_{I} \subseteq\{f \mid f \leq B \quad \& \quad f \leq C\}$.

Let $\left\langle\phi_{i}\right\rangle_{i \in \omega}$ be an enumeration of all eligible sentences. As a relation between $i$ and $n$, " $n$ codes a condition forcing $\phi_{i}$ " is recursive in $f$. Let $P_{0}=Q_{0}=$ id. Let $\left(P_{2 i+1}, Q_{2 i+1}\right)=$ the condition with least code which extends $\left(P_{2 i+1}, Q_{2 i+1}\right)$ such that $(f)_{i} \leq P_{2 i+2} \&(f)_{i} \leq Q_{2 i+2}$. By [3, Lemma 3.3] such a condition exists; let $h(2 i+2)=$ this least code. The even stages insure the truth of (ii). (ii) and the genericity of the sequence insure that $(\underline{b}, \underline{c})$ is exact for $I$, where $\underline{b}=\operatorname{deg}(B)$, $\underline{c}=\operatorname{deg}(C)$. To decide whether $n \in(B \oplus C)^{(2)}$, find $i$ such that $\phi_{i}=$ " $\underline{n} \in(\underline{B} \oplus \underline{C})^{(2)}$ ", and ask $f$ whether the condition with code $h(2 i+1)$ forces $\phi_{i}$.

$(1) \Rightarrow\left(3^{\prime}\right)$ Let a condition be a finite sequence of graphs of members of $M_{I}$. If $M$ and $N$ are conditions, $M$ extends $N$ iff $N$ is an initial segment of $M$. If $M=\left(C_{0}, \ldots, C_{n-1}\right), \operatorname{lh}(M)=n, M^{\cap} C=\left(C_{0}, \ldots, C_{n-1}, C\right), M^{*}=\{\langle\langle i, x\rangle, y\rangle \mid i<$ $\left.n \&\langle x, y\rangle \in C_{i}\right\}$. We construct a sequence $\left\langle M_{n}\right\rangle_{n \in \omega}$ of conditions and a $g$ such that $\operatorname{graph}(g)=\bigcup_{n}\left\{M_{n}^{*}\right\}, g$ parametrizes $M_{I}$ and $g^{\prime} \leq f$. We will also provide an $h \leq f$ such that $(f)_{h n}=\operatorname{graph}\left(M_{n}^{*}\right)$.

Let $\sigma$ be single-valued iff $\hat{\sigma}$ is the graph of a partial function; let $\sigma$ be compatible with $M$ iff $(\forall i)$ (if $i \in \hat{\sigma} \& l(l(i))<\operatorname{lh}(M)$ then $i \in M^{*}$ ).

Let $M_{0}=$ the empty sequence; $(f)_{h(0)}=\operatorname{graph}(\varnothing)$. At stage $2 i+1$ we use a technique of Friedberg $\left[5\right.$, p. 265] to insure that $g^{\prime} \leq f$ : we try to insure that $i \in g^{\prime}$. If

$(\exists \sigma)\left(\sigma\right.$ is compatible with $M_{2 i} \& \sigma$ is single-valued \& $\{i\}^{*^{\sigma}}(i)$ converges $)$,

select a specific such string $\sigma$. Let $m=\max \{l(l(x)) \mid x \in \hat{\sigma}\}$. Let $M_{2 i+1}$ be that condition $M$ extending $M_{2 i}$ such that $\hat{\sigma} \subseteq M^{*}$ and such that for any $x$ such that $\operatorname{lh}\left(M_{2 i}\right) \leq l(l(x))<m$ and $x \notin \hat{\sigma}, x \in M^{*}$ iff $r(x)=0$. If $\left(1_{i}\right)$ is false, let $M_{2 i+1}=M_{2 i}$. Let $M_{2 i+2}=M_{2 i+1}{ }^{n} \operatorname{graph}\left((f)_{i}\right)$. Clearly $g$ parametrizes $M_{I}$ by these even steps. $\left(1_{i}\right)$ is decidable in $(f)_{h(2 i)}^{\prime}$; an $f$-index for the latter may be located from $h(2 i)$ by the niceness of $f$. If $\left(1_{i}\right)$ is true, the specific string chosen at stage $2 i+1$ may be found recursively in $(f)_{h(2 i)}$. Thus $h(2 i+1)$ may be computed in $f$. Similarly, $h(2 i+2)$ is computable in $f$ from $h(2 i+1)$. Finally, $i \in g^{\prime}$ iff $\left(1_{i}\right)$ is true. Thus $g^{\prime} \leq f$.

$(1) \Rightarrow\left(4^{\prime}\right)$ This argument exploits the forcing aspect of the previous argument. Identifying sets and characteristic functions, let a condition be a finite sequence of members of $M_{I} \cap 2^{\omega}$. If $M$ is a condition, let $\operatorname{lh}(M)$ and $M^{\cap} B$ be defined as above; let

$$
M^{*}=\left\{\langle i, x+1\rangle \mid i<n \& x \in C_{i}\right\} \cup\{\langle i, 0\rangle \mid i<n\},
$$

where $M=\left(C_{0}, \ldots, C_{n-1}\right)$. The peculiarity of this definition is to insure that 
$\operatorname{lh}(M)$ is determined by $M^{*}$, even if a final segment of $M$ consists of entries of $\varnothing$. Let $f \in \underline{a}$ nicely parametrize $M_{I}$. Let $n$ code a condition $M$ iff $(f)_{n}=M^{*}$. We construct a sequence $\left\langle M_{n}\right\rangle_{n \in \omega}$ and define $A$ by: $\langle i, x\rangle \in A$ iff $\langle i, x+1\rangle \in$ $\bigcup_{n}\left\{M_{n}^{*}\right\} ; A$ shall parametrize $M_{I} \cap 2^{\omega}$ and $A^{(2)} \leq f$. Again, we will provide an $h \leq f$ such that $h(n)$ codes $M_{n}$.

Let $\sigma$ be compatible with $M$ iff for some condition $N$ extending $M, \hat{\sigma} \subseteq N^{*}$. Form a forcing language as in the proof of $(1) \Rightarrow\left(2^{\prime}\right)$, except with only the generic constant " $\underline{B}$ ". Define forcing as a relation between conditions and sentences in $\Pi_{0}^{0} \cup \Sigma_{1}^{0} \cup \Pi_{1}^{0}$, the eligible sentences, as follows:

$\begin{array}{ll}M \Vdash " \underline{B}(\underline{n}) " & \text { iff }(\exists \sigma)\left(\sigma \in \operatorname{Str} \& \sigma \subseteq M^{*} \&\{n\} *^{*^{\sigma}}(n) \operatorname{con}\right. \\ M \Vdash \phi & \text { iff } \phi \text { is true, where } \phi \text { is atomic and } \\ & \text { not of the form “ } \underline{B}(\underline{n}) " ; \\ M \Vdash(\exists x) \phi x & \text { iff }(\exists n) M \Vdash \phi(\underline{n}) ; \\ M \Vdash-\phi & \text { iff }(\forall N) \text { (if } N \text { extends } M \text { then } M \Vdash \phi) .\end{array}$

$M \Vdash-\underline{B}(\underline{n})$ iff $(\forall \sigma)$ (if $\sigma \in \operatorname{Str} \& \sigma$ is compatible with,$M$ then $\{n\}^{*^{\sigma}}(n)$ diverges). Because " $\sigma$ is compatible with $M$ " is recursive in $M$, " $M \Vdash$ $-\underline{B}(\underline{n})$ " is $\Pi_{1}^{0}$ in $M^{*}$. Furthermore, " $n$ codes a condition" and " $n$ codes a condition extending the one $m$ codes" are $\Sigma_{2}^{0}$ in $(f)_{n}$ and $(f)_{n} \oplus(f)_{m}$. For $\phi \in \Pi_{0}^{0} \cup \Sigma_{1}^{0}$, “ $M \Vdash \phi$ " is $\Sigma_{1}^{0}$ in $M^{*}$; thus " $n$ codes a condition forcing $\phi$ " is decidable in $f$. The statement " $M \Vdash \phi$ " for $\phi \in \Pi_{1}^{0}$, has the form

$$
\left(\forall X \in M_{I} \cap 2^{\omega}\right)(\forall x)(\exists y) Q\left(\phi, x, y, X, M^{*}\right),
$$

$Q$ recursive. This is equivalent to: $\left(\forall X \leq\left(M^{*}\right)^{\prime}\right)(\forall x)(\exists y) Q\left(\phi, x, y, X, M^{*}\right)$. To see this, suppose that $\left(\exists X \in M_{I} \cap 2^{\omega}\right)(\exists x)(\forall y)-Q(\cdots)$. Thus for some $n$, $(\exists X)(\forall y)-Q(\cdots \underline{n} \cdots)$. By Kreisel's basis theorem [6, p. 187], $(\exists X \leq$ $\left.\left(M^{*}\right)^{\prime}\right)(\forall y)-Q(\cdots \underline{n} \cdots)$, giving the nontrivial part of the equivalence. Thus “ $M \Vdash \phi$ ”, for $\phi \in \Pi_{1}^{0}$ is $\Sigma_{3}^{0}$ in $\left(M^{*}\right)^{\prime}$. Thus for any $\phi \in \Pi_{0}^{0} \cup \Sigma_{1}^{0} \cup \Pi_{1}^{0}$, " $n$ codes a condition forcing $\phi$ " is decidable in $f$.

An $\omega$-sequence of conditions is generic iff for each $n$, the $n+1$ st extends the $n$ th, and each eligible sentence is decided by some member of the sequence. Fix an enumeration $\left\langle\phi_{i}\right\rangle_{i \in \omega}$ of the eligible sentences. Fix $g \leq f$ such that $(f)_{g(n)}=\left\{x \mid(f)_{n}(x) \neq 0\right\}$. Let $M_{0}=$ the empty sequence with code $h(0)$. Let $M_{2 i+1}=$ the condition with least code which extends $M_{2 i}$ and decides $\phi_{i}$; let $h(2 i+1)$ be this least code. By the previous remarks, $h(2 i+1)$ may be computed from $f$. Let $M_{2 i+2}=M_{2 i+1}{ }^{n}(f)_{g(i)}$; let $h(2 i+2)$ be a code for $M_{2 i+2}$; again, it may be computed from $f$. The even stages insure that $A$, defined two paragraphs back, parametrizes $M_{I} \cap 2^{\omega}$.

By the definition of forcing, " $\underline{B}(\underline{i})$ " is forced by a condition in this generic sequence iff $i \in\left(\bigcup_{n}\left\{M_{n}^{*}\right\}\right)^{\prime} ; "$ " $-\underline{B}(\underline{i})$ " is forced iff $i \notin\left(\bigcup_{n}\left\{M_{n}^{*}\right\}\right)^{\prime}$. Letting $B$ be the extension of " $\underline{B}$ " determined by the generic sequence, $B=\left(\cup_{n}\left\{M_{n}^{*}\right\}\right)$ '. To decide whether $i \in\left(\bigcup_{n}\left\{M_{n}^{*}\right\}\right)^{(2)}$, find $j$ such that $\phi_{j}=$ " $\underline{i} \in \underline{B}$ " " and ask $f$ whether the condition with code $h(2 j+1)$ forces $\phi_{j}$. Clearly $A \leq \bigcup_{n}\left\{M_{n}^{*}\right\}$. Thus $A^{(2)} \leq f$. 
$\left(3^{\prime}\right) \Rightarrow(3)$ Let $\underline{b}$ be a u.u.b. on $I$ such that $\underline{b}^{\prime} \leq \underline{a}$. By the Friedberg completeness theorem relative to $\underline{b}$, there is a $\underline{c} \geq \underline{b}, \underline{c}^{\prime}=\underline{a}$. By observation (4), $\underline{c}$ is a u.u.b. on $I$.

$\left(4^{\prime}\right) \Rightarrow(4)$ Let $\underline{b}$ be a weak u.u.b. on $I, \underline{b}^{(2)} \leq \underline{a}$. As above, there is a $\underline{c} \geq \underline{b}$ such that $\underline{c}^{(2)}=\underline{a}$. Again, $\underline{c}$ is a weak u.u.b. on $I$.

$\left(2^{\prime}\right) \Rightarrow(2)$ The following argument is due to Carl Jockusch. It suffices to prove that:

$$
\begin{aligned}
& (\forall \underline{b})(\forall \underline{c})(\forall \underline{d})\left((\underline{b} \cup \underline{c})^{\prime} \leq \underline{d}\right. \\
& \Rightarrow\left(\exists \underline{b}^{*}\right)\left(\exists \underline{c}^{*}\right)\left(\underline{b}^{*} \geq \underline{b} \& \underline{c}^{*} \geq \underline{c}\right. \\
& \left.\left.\& J_{\underline{b}} \cap J_{\underline{c}}=J_{\underline{b}^{*}} \cap J_{\underline{c}^{*}} \&\left(\underline{b}^{*} \cup \underline{c}^{*}\right)^{\prime}=\underline{d}\right)\right) .
\end{aligned}
$$

Suppose $(\underline{b} \cup \underline{c})^{(2)} \leq \underline{a}$. By the Friedberg completeness theorem relativized to $(\underline{b} \cup \underline{c})^{\prime}$, choose $\underline{d} \geq(\underline{b} \cup \underline{c})^{\prime}$ such that $\underline{a}=\underline{d}^{\prime}$. By $(\mathrm{J})$, there are $\underline{b}^{*}$ and $\underline{c}^{*}$, $\underline{b}^{*} \geq \underline{b}, \quad \underline{c}^{*} \geq \underline{c}, \quad$ such that $J_{\underline{b}} \cap J_{\underline{c}}=J_{\underline{b}^{*}} \cap J_{\underline{c}^{*}}$ and $\left(\underline{b}^{*} \cup \underline{c}^{*}\right)^{\prime}=d$. Thus $\left(\underline{b}^{*} \cup \underline{c}^{*}\right)^{(2)}=\underline{a}$.

We sketch Jockusch's proof of (J). Let $B \in \underline{b}, C \in \underline{c}, D \in \underline{d}$. We will construct $B^{*}$ and $C^{*}$ and let $\underline{b}^{*}=\operatorname{deg}\left(B \oplus B^{*}\right), \underline{c}^{*}=\operatorname{deg}\left(C \oplus C^{*}\right)$. We will let $B^{*}=\bigcup_{i}\left\{\hat{\sigma}_{i}\right\}, C^{*}=\bigcup_{i}\left\{\hat{\tau}_{i}\right\}$. Let $\sigma_{0}=\tau_{0}=$ the empty string.

Stage $3\langle k, i\rangle+1$. We satisfy the requirement: if $\{k\}^{B \oplus B^{*}}=\{i\}^{C \oplus C^{*}}$ and they are total, then $\{k\}^{B \oplus B^{*}} \leq B$ and $\{i\}^{C \oplus C^{*}} \leq C$. If there are $\sigma \supseteq \sigma_{3(k, i)}, \tau \supseteq \tau_{3(k, i)}$ and $x$ such that $\{k\}^{*^{B \oplus \sigma}}(x)$ and $\{i\}^{*^{C \oplus r}}(x)$ are defined and distinct, let $\sigma_{3(k, i)+1}$ and $\tau_{3(k, i)+1}$ be the least such $\sigma$ and $\tau$; otherwise let $\sigma_{3(k, i)+1}=\sigma_{3(k, i\rangle}, \tau_{3\langle k, i\rangle+1}=$ $\tau_{3\langle k, i\rangle}$. In the former case, for any $B^{*}$ and $C^{*}$ such that $\sigma_{3(k, i)+1} \subseteq B^{*}$ and $\tau_{3(k, i)+1} \subseteq C^{*}$, the requirement is vacuously satisfied. In the latter case, to compute $\{k\}^{B \oplus B^{*}}(x)$ for any such $B^{*}$, search for $\sigma \supseteq \sigma_{3 k, i\rangle}$ such that $\{k\}^{* B \oplus \sigma}(x)$ is defined and output the result. Similarly for any such $C^{*}$. Thus the requirement is satisfied.

Stage $3 j+2$. If there are $\sigma$ and $\tau, \sigma \supseteq \sigma_{3 j+1}$ and $\tau \supseteq \tau_{3 j+1}$, such that $\{j\}^{*(A \oplus \sigma) \oplus(\boldsymbol{B} \oplus \sigma)}(j)$ converges, let $\sigma_{3 j+2}$ and $\tau_{3 j+2}$ be the least such $\sigma$ and $\tau$. Otherwise let $\sigma_{3 j+2}=\sigma_{3 j+1}, \tau_{3 j+2}=\tau_{3 j+1}$.

Stage $3 j+3$. Let $\sigma_{3 j+3}=\sigma_{3 j+2}{ }^{n} D(j), \tau_{3 j+3}=\tau_{3 j+2}{ }^{n} D(j)$.

Stages $3 j+1$ and $3 j+2$ can be carried out effectively in $(B \oplus C)^{\prime}$; stages $3 j+3$ can be carried out effectively in $D$. The stages $3 j+2$ insure that $\left(\left(B \oplus B^{*}\right) \oplus\left(C \oplus C^{*}\right)\right)^{\prime} \leq D$. Because $(B \oplus C)^{\prime} \leq\left(\left(B \oplus B^{*}\right) \oplus\left(C \oplus C^{*}\right)\right)^{\prime}$, stages $3 j+1$ and $3 j+2$ can be carried out effectively in $\left(\left(B \oplus B^{*}\right) \oplus\right.$ $\left.\left(C \oplus C^{*}\right)\right)^{\prime}$. Thus we could carry out stages $3 j+3$ effectively in $\left(\left(B \oplus B^{*}\right) \oplus\right.$ $\left.\left(C \oplus C^{*}\right)\right)^{\prime}$, and thereby recover $D$. If $f \leq B$ and $f \leq C$, clearly $f \leq\left(B \oplus B^{*}\right)$ and $f \leq\left(C \oplus C^{*}\right)$. The converse has been insured by stages $3 i+1$. QED

Corollary. If $M_{I}=$ the arithmetic functions, I has a weak u.u.b. which is not a u.u.b.

Proof. $\underline{Q}^{(\omega)}$ is a nice u.u.b. on $I$. Suppose $\underline{0}^{(\omega)}=\underline{a}^{(2)}$ for $\underline{a}$ a weak u.u.b. on $I$. If $\underline{a}$ is also a u.u.b. on $I, \underline{Q}^{(\omega)} \leq \underline{a}^{\prime}$, because $\underline{0}^{(\omega)}$ is recursive in the jump of any u.u.b. on $I($ see $[3, \S 6])$. Contradiction. This answers a question raised in $[3, \S 6]$.

COROLlaRY. The relation "I is an ideal and a is the jump of a u.u.b. on I" is first-order definable over $\left\langle D, \leq,{ }^{\prime}\right\rangle$. 
The above theorem leaves open the important question: Is " $I$ is an ideal and $\underline{a}$ is a u.u.b. on $I$ " first-order definable over that structure?

§3. Systems of notation. The motivation for this paper is hierarchy theoretic. Thus, as is traditional, we shall devote some attention to systems of notation. Relations shall be identified with sets of natural numbers via the pairing function, and thus with characteristic functions. We prefer reflexive relations because if $R$ is reflexive, $\operatorname{Fld}(R) \leq R$. A reflexive relation is wellfounded iff no strictly decreasing function is an infinite descending path through that relation. If $R$ is wellfounded, let $|x|_{R}=$ the height in $R$ of $x$, $|R|=$ the height of $R ; x<_{R} y$ iff $x R y \& x \neq y ; R_{x}=\{\langle y, z\rangle \mid\langle y, z\rangle \in R \&$ $\left.z<_{R} x\right\}$.

Suppose $R$ is a reflexive wellfounded relation, $|R|$ is a limit ordinal, and each $x \in \operatorname{Fld}(R)$ has at most one predecessor under $R$. Let

$$
\begin{aligned}
& H_{x}(R)=\left\{\begin{array}{l}
\omega \quad \text { if }|x|_{R}=0 \\
H_{y}(R)^{\prime} \quad \text { if } x \text { is the } R \text {-successor of } y ; \\
\left\{\langle y, z\rangle \mid z \in H_{y}(R) \& y<_{R} x\right\} \text { if } x \text { is an } R \text {-limit. }
\end{array}\right. \\
& H(R)=\left\{\langle x, z\rangle \mid x \in \operatorname{Fld}(R) \& z \in H_{x}(R)\right\} .
\end{aligned}
$$

These definitions relativize to any $A \subseteq \omega$, by letting $H_{x}(R)^{A}=A$, if $|x|_{R}=0$. All of the following definitions may also be so relativized. Let $R$ have the uniqueness property iff for any $x$ and $y$ in $\operatorname{Fld}(R)$ such that $|x|_{R}=|y|_{R}$, $\operatorname{deg}\left(H_{x}(R)\right)=\operatorname{deg}\left(H_{y}(R)\right)$. Suppose $R$ has the uniqueness property. Let $h_{\alpha}(R)=\operatorname{deg}\left(H_{x}(R)\right)$, for $|x|_{R}=\alpha ; h_{|R|}(R)=h(R)=\operatorname{deg}(H(R))$. For $\lambda \leq|R|$, $\lambda$ a limit ordinal, let $I_{\lambda}(R)$ be the minimal ideal containing $\left\{h_{\alpha}(R) \mid \alpha<\lambda\right\}$. Then $h_{\lambda}(R)$ is a u.u.b. on $I_{\lambda}(R)$.

$R$ is a system of notation (hereafter an s.n.) iff $R$-successors are unique and there are functions $s_{R} \leq R$ and $f \leq R$ such that for any $x \in \operatorname{Fld}(R): f(x)=0$ if $|x|_{R}=0 ; f(x)=1$ if $|x|_{R}$ is a successor; $f(x)=2$ if $|x|_{R}$ is a limit; $s_{R}(x)$ is the $R$-successor of $x$.

Observation. (5) If $R$ is a s.n. then there are $f$ and $g, f \leq H(R), g \leq H(R)$, such that $f$ parametrizes $M_{I_{|R|}(R)}$ and $g$ is an $f$-jump locator.

Let $R$ have the uniform uniqueness property (hereafter the u.u. property) iff for some recursive $g$, for any $x$ and $y$ such that $|x|_{R} \leq|y|_{R}, H_{x}(R)=$ $\{g(x, y)\}^{H_{y}(R)}$.

Observations. (6) If $R$ has the u.u. property, there are $f$ and $g, f \leq H(R)$ and $g \leq H(R)$, such that $f$ parametrizes $M_{I_{|R|}(R)}$ and $g$ is an $f$-join locator.

(7) If $R$ is a linear s.n., $R$ has the u.u. property.

Let $R$ have the $I$-initial segment property iff for every $x, R_{x} \in M_{I}$.

Hereafter, identify the hyperjump of $f$ with $W^{f}=\left\{x \mid\{x\}^{f}\right.$ is a wellordering $\}$. We distinguish three sorts of $A$-hierarchy ideals. $I$ is a case 1 ideal iff $M_{I}=L_{\alpha}[A] \cap \omega^{\omega}$ where for some $B \in M_{I}, \alpha<\omega_{1}^{B}$. I is a case 2 ideal iff $M_{I}=L_{\alpha}[A] \cap \omega^{\omega}$, where for some $B \in M_{I}, \alpha=\omega_{1}^{B} . I$ is a case 3 ideal iff $I$ is an $A$-hierarchy ideal under neither case 1 nor case 2 . If $I$ is a case 1 or a case 2 ideal, let $\operatorname{ht}(I)=\omega \alpha$, for $\alpha$ as in the above definition. If $I$ is a case 3 ideal, 
$\mathrm{ht}(I)=\sup \left\{|S| \mid S\right.$ is wellfounded \& $\left.S \in M_{I}\right\}$.

THEOREM 2. Let I be an A-hierarchy ideal falling under case 1 or case 2. The following are equivalent:

(1) $\underline{a}$ is a nice u.u.b. on I;

(5) for some $R$, a linear s.n. with the I-initial segment property, $|R|=\mathrm{ht}(I) \&$ $\underline{a}=h(R)^{A}$.

Proof. We omit mention of $A$ where possible.

$(5) \Rightarrow$ (1) By observations (5) and (6), it suffices to show that $I=I_{|R|}(R)$. Using the $I$-initial segment property, this is an easy exercise in $L$-manship. For $(1) \Rightarrow(5)$, it suffices to prove that (1) implies:

(5') For some $R$, a linear s.n. with the $I$-initial segment property, $|R|=\mathrm{ht}(I)$ $\& h(R) \leq \underline{a}$;

and then show that $\left(5^{\prime}\right) \Rightarrow(5)$.

$\left(5^{\prime}\right) \Rightarrow(5)$ Let $B \in \underline{a}$. If $|R|$ is not a limit of limits, choose $x_{0}$ such that $\left|x_{0}\right|_{R}+\omega=|R|$. For $x<_{R} x_{0}$, let $x^{*}=\langle 0, x\rangle$; for $x$ such that $x_{0} R x$, let $x^{*}=$ $\langle 1,\langle n, x\rangle\rangle$ if $n \in B, x^{*}=\langle 2,\langle n, x\rangle\rangle$ otherwise. Define $S$ by $x^{*} S y^{*}$ iff $x R y . R \leq S$ and $B \leq S$; thus $s_{S} \leq(R \oplus B) \leq S$. $S$ has the $I$-initial segment property. Finally, $H(S) \equiv(H(R) \oplus B)$. If $|R|$ is a limit of limits, there is a $g \leq R$ enumerating an $R$-increasing sequence of $R$-limits cofinal with $R$. For $x \notin \operatorname{Rng}(g)$, let $x^{*}=\langle 0, x\rangle$; for $x=g(n)$, let $x^{*}=\langle 1,\langle n, x\rangle\rangle$ if $n \in B, x^{*}=$ $\langle 2,\langle n, x\rangle\rangle$ otherwise. Define $S$ as above. Notice that $\operatorname{Rng}(g) \leq R$. As above, $S$ is a linear s.n. with the $I$-initial segment property, and $H(S) \equiv(H(R) \oplus B)$.

$(1) \Rightarrow\left(5^{\prime}\right)$ Suppose $I$ is an ideal falling under case 1: for $C \in M_{I}, M_{I}=$ $L_{\alpha}[A] \cap \omega^{\omega}, \alpha<\omega_{1}^{C}$. Suppose $C \in L_{\lambda+1}[A]-L_{\lambda}[A]$. By well-known results about $L, C^{\prime}$ computes a linear s.n. $R$, such that $|R|=\lambda$, and each $R_{x}$ belongs to $L_{\lambda}[A]$. Furthermore, $H(R) \in L_{\lambda+1}[A]$. Let $S$ be a linear s.n. recursive in $C$, $|S|=\alpha-\lambda$. Form a linear s.n. by effectively "tagging" a copy of $S$ after a copy of $R$; call the result $T$. By an easy generalization of Corollary 6.2 of [3], $h(T)$ is recursive in the jump of any u.u.b. on $I$, and thus, by Theorem 1 , in any nice u.u.b. on $I$.

Suppose $I$ is a case 2 ideal. Say $\mathrm{ht}(I)=\omega_{1}^{C}$ for $C \in M_{\mathrm{I}}$. Let $B=\left\{i \mid\{i\}^{C}\right.$ is a wellordering without a last element $\&(\forall x)$ (if $x \in \operatorname{Fld}\left(\{i\}^{C}\right)$ then $2^{x}$ is the $\{i\}^{C}$-successor of $\left.\left.x\right)\right\} . B$ is $\Sigma_{1}^{1}$ over $M_{I}$. Let $f \in \underline{a}$ nicely parametrize $M_{I}$, where $\underline{a}$ is a given nice u.u.b. on $I$. $B$ is $\Sigma_{1}^{0}$ in $f$. Let $g \leq f$ enumerate $B$. Define $R$ by:

$$
\begin{gathered}
\langle n, x\rangle R\langle m, y\rangle \text { iff }\left(n<m \& x \in \operatorname{Fld}\left(\{g(n)\}^{C}\right) \& y \in \operatorname{Fld}\left(\{g(m)\}^{C}\right)\right) \\
\text { or }\left(n=m \&\{g(n)\}^{C}(\langle x, y\rangle)=1\right) .
\end{gathered}
$$

$R$ is a linear s.n. and $|R|=\omega_{1}^{C}$. Furthermore, $R$ has the $I$-initial segment property. There is a $g^{*} \leq f$ such that $(f)_{g^{*}(x)}=R_{x} . H(R)$ is $\Delta_{1}^{1}$ over $M_{I}$. Using $g^{*}, H(R) \leq f . \quad$ QED

Curiously enough, Theorem 2 does not extend to ideals falling under case 3 . If $f$ parametrizes $M_{I}$ for such an ideal $I$, define " $f$-hyperjump locator" and "hypernice u.u.b. on $I$ " by replacing all references to the jump operation in Definitions 5 and 6 by references to the hyperjump operation.

THEOREM 3. Let I be a case 3 A-hierarchy ideal. The following are equivalent: 
$\left(1^{*}\right) \underline{a}$ is a hypernice u.u.b. on $I$;

$\left(2^{*}\right)$ for some $\underline{b}$ and $\underline{c},(\underline{b}, \underline{c})$ is an exact pair for $I \& \underline{a}=(\underline{b} \cup \underline{c})^{(3)}$;

$\left(^{*}\right)$ for some $\underline{b}, \underline{b}$ is $a$ u.u.b. on $I \& \underline{a}=\underline{b}^{(2)}$;

$\left(4^{*}\right)$ for some $\underline{b}, \underline{b}$ is a weak u.u.b. on $I \& \underline{a}=\underline{b}^{(3)}$;

$\left(5^{*}\right)$ for some linear s.n. $R$ with the I-initial segment property, $|R|=\mathrm{ht}(I) \&$ $\underline{a}=h(R)^{A}$.

Clearly this theorem implies the existence of a nice u.u.b. $\underline{a}$ for which $\left(5^{*}\right)$ fails. The proof of this theorem is a routine extension of the proof of Theorem 1 , except for $\left(1^{*}\right) \Leftrightarrow\left(5^{*}\right)$.

$\left(1^{*}\right) \Rightarrow\left(5^{*}\right)$ Let $B=\left\{n \mid(f)_{n}\right.$ is a wellordering without a last element and with successor function $\left.\lambda x \cdot 2^{x}\right\}$. By $\left(1^{*}\right), B \leq f$, where $f \in \underline{a}$ hypernicely parametrizes $M_{\mathrm{I}}$. Define $R$ by

$$
\begin{aligned}
\langle n, x\rangle R\langle m, y\rangle & \operatorname{iff}\left(\left(n<m \& x \in \operatorname{Fld}\left((f)_{n}\right) \& y \in \operatorname{Fld}\left((f)_{m}\right)\right)\right. \\
& \text { or } \left.\left(n=m \&(f)_{n}(\langle x, y\rangle)=1\right)\right) \& n \in B \& m \in B .
\end{aligned}
$$

From here, the argument parallels that of Theorem $2,(1) \Rightarrow\left(5^{\prime}\right)$, case 2 . We end up with $h(R) \leq \underline{a}$. Clearly $R$ has the $I$-initial segment property. The technique used in proving Theorem $2,\left(5^{\prime}\right) \Rightarrow(5)$, will now prove $\left(5^{*}\right)$.

$\left(5^{*}\right) \Rightarrow\left(1^{*}\right)$ Let $R$ be a witness for $\left(5^{*}\right)$. By the proof of Theorem 2 , $(5) \Rightarrow(1), h(R)$ is a nice u.u.b. on $I$. Let $f \in h(R)$ nicely parametrize $M_{I}$. Suppose $g \in M_{\mathrm{I}} .|x|_{R} \geq \omega_{1}^{g}$ iff:

$(\forall i)$ ((if $\{i\}^{g}$ is well-founded then $(\exists h)$ ( $h$ maps $\operatorname{FLD}\left(\{i\}^{g}\right)$ order preserving into $\left.R_{x}\right)$ ) \& (if $\{i\}^{g}$ is not wellfounded then $(\exists h)\left(h\right.$ is an infinite descending path through $\left.\left.\{i\}^{g}\right)\right)$ ),

which is equivalent to:

$(\forall i)\left((\exists h)\left(h\right.\right.$ maps Fld $\left(\{i\}^{g}\right)$ order preserving into $\left.R_{x}\right)$ or $(\exists h)$ ( $h$ is an infinite descending path through $\left.\{i\}^{g}\right)$ ).

If $|x|_{R} \geq \omega_{1}^{g}$ and $\{i\}^{g}$ is wellfounded, then there is an $h$ mapping $\operatorname{Fld}\left(\{i\}^{g}\right)$ order preserving into $R_{x} ; h \leq H_{x}(R)$, using the fact that $R$ has the $I$-initial segment property. Thus the first quantifier on " $h$ " in (*) and (**) may be restricted by " $h \leq H_{x}(R)$ ". If $|x|_{R} \geq \omega_{1}^{g}, H_{x}(R)$ computes a nice parametrization of $\mathrm{HYP}(g) ; W^{g} \in \Sigma_{1}^{1}(\mathrm{HYP}(g))$; so $W^{g} \leq H_{s_{R} x}(R)$. If $\{i\}^{g}$ is not wellfounded, some infinite descending path through $\{i\}^{g}$ is recursive in $W^{g}$. Thus the second quantifier on " $h$ " in $(*)$ and $(* *)$ may be restricted by " $h \leq H_{s_{R} x}(R)$ " preserving truth. Given $x, f$-indices for $R_{x}, H_{x}(R)$ and $H_{s_{R} x}(R)$ may be computed in $H(R)$ and thus in $f$. By these remarks, given an $f$-index for $g$, " $|x|_{R} \geq \omega_{1}^{g}$ ?" is decidable in $f$. If $|x|_{R} \geq \omega_{1}^{g},(f)_{m}=W^{(f)_{n}}$ iff:

$(\forall i)\left(\left((\exists j)\left(\{j\}^{H_{s_{R^{x}}}(R)}\right.\right.\right.$ is an infinite descending path through $\left.\{i\}^{(f)_{n}} \Rightarrow(f)_{m}(i)=0\right) \&\left((\exists j)\left(\{j\}^{H_{x}(R)}\right.\right.$ maps Fld $\left(\{i\}^{(f)_{n}}\right)$ order preserving into $\left.\left.R_{x}\right) \Rightarrow(f)_{m}(i)=1\right)$ ).

This too is decidable in $f$. To find an $m$ such that $(f)_{m}=W^{(f)_{n}}$, first search and 
find an $x$ such that $|x|_{R} \geq \omega_{1}^{(f)_{n}}$; then search and find the desired $m$. QED

§4. Leastness questions. As mentioned, any case 1 ideal has a least nice u.u.b. What follows is a negative answer to all other relevant leastness questions.

THEOREM 4. Let I be a countable ideal. If $\underline{a} \notin$ I then there is $a \underline{b}, \underline{b} a$ u.u.b. on I, such that $\underline{a} \not \underline{b}$.

THEOREM 5. Let I be a case 2 or a case 3 hierarchy ideal. If $\underline{a} \notin$ I then there is $a \underline{b}, \underline{b}$ is a nice u.u.b. on $I$, such that $\underline{a} \not \underline{b}$.

PROOF OF THEOREM 4. The terminology is as in Theorem $1,(1) \Rightarrow\left(3^{\prime}\right)$. If $M$ is a condition and $\sigma$ is compatible with $M$, we define $\{i\}^{*^{M \cdot} \cup \sigma}(n)$ by viewing $M^{*} \cup \sigma$ as a partial object; in other words, it is $\{i\}^{M^{*} \cup \hat{\sigma}}(n)$ provided all questions " $x \in M^{*} \cup \hat{\sigma}$ ?" asked of the oracle are for $x$ such that $l(l(x))<$ $\operatorname{lh}(M)$ or $x<\operatorname{lh}(\sigma)$; otherwise it diverges. Given $f \in \underline{a}$, we construct a parametrization $g$ such that $f \not g$. Fix an enumeration $\left\langle h_{i}\right\rangle_{i \in \omega}$ of $M_{I}$. Let $M_{0}=$ the empty sequence.

Stage $2 i+1$. We find the least $\langle\sigma, x\rangle$ such that $\sigma$ is compatible with $M_{2 i}$, single-valued, and either

$(\forall \tau)$ (if $\sigma \cup \tau$ is single-valued \& compatible with $M_{2 i}$ then

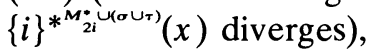

or

$$
\{i\}^{\text {M }_{2 i}^{*}{ }^{\circ \sigma}}(x)=k, \text { for some } k \neq f(x) .
$$

Claim. There are such $\sigma$ and $x$. Otherwise for any $\sigma$ and $x$, if $\sigma$ is single-valued and compatible with $M_{2 i},\left(2_{i}\right)$ and $\left(3_{i}\right)$ are false. Pick $\tau_{0}$ singlevalued and compatible with $M_{2 i}$ such that $\{i\}^{*_{2 i} v_{0} \tau_{0}}(0)$ converges; with $\tau_{n}$ already chosen, pick $\tau_{n+1}$ extending $\tau_{n}$, single-valued and compatible with $M_{2 i}$,

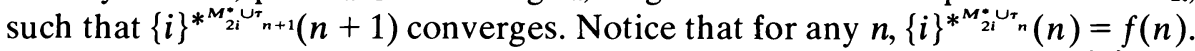
We can enumerate such a sequence recursively in $M_{2 i}^{*}$. Let $A=M_{2 i}^{*} \cup \bigcup_{i}\left\{\tau_{j}\right\}$. Thus $f=\{i\}^{A}$, and $A \leq\left(M_{2 i}^{*}\right)^{\prime} \in M_{\mathrm{I}}$. This contradicts our choice of $f$.

Let $M_{2 i+1}=M_{2 i} \cap \operatorname{graph}\left(f_{1}\right)^{n} \cdots \cap \operatorname{graph}\left(f_{m}\right)$, where for $j \leq m: f_{i}(x)=y$ iff either $\langle\langle k+j, x\rangle, y\rangle \in \hat{\sigma}$ or $-(\exists v)(\langle\langle k+j, x\rangle, v\rangle \in \hat{\sigma})$ but $y=0$, where $m=$ $\max \{l(l(x)) \mid x \in \hat{\sigma}\}, k=\operatorname{lh}\left(M_{2 i}\right)$. Let $M_{2 i+2}=M_{2 i+1}{ }^{n} \operatorname{graph}\left(h_{i}\right)$. Let $g$ be such that graph $(g)=\bigcup_{i}\left\{M_{i}^{*}\right\}$. The even stages insure that $g$ parametrizes $M_{I}$. Stage $2 i+1$ insures that $f \neq\{i\}^{8}$. QED

Proof of TheOREm $5, I$ Under Case 2 . Given $f \in \underline{a}$, we will construct sets $B$ and $C$ such that $(\operatorname{deg}(B), \operatorname{deg}(C))$ is an exact pair for $I$ and $f \not \nexists(B \oplus C)^{(2)}$. By Theorem $1, \operatorname{deg}\left((B \oplus C)^{(2)}\right)$ is the desired nice u.u.b. We return to the forcing situation of Theorem $1,(1) \Rightarrow\left(2^{\prime}\right)$, except that now we extend the class of eligible sentences to include $\Sigma_{3}^{0} \cup \Pi_{3}^{0}$ :

$$
(P, Q) \Vdash(\exists x) \phi \quad \text { iff }(\exists n)((P, Q) \Vdash \phi \underline{n}),
$$

where $\phi \in \Pi_{2}^{0}$; 


$$
(P, Q) \Vdash-\phi \quad \text { iff }(\forall(R, S)) \text { (if }(R, S) \text { extends }(P, Q) \text { then }
$$

$$
(R, S) \Vdash \phi) \text {, where } \phi \in \Sigma_{3}^{0} \text {. }
$$

We construct a generic sequence of conditions with limit $(B, C)$ such that $M_{I} \subseteq\{f \mid f \leq B \& f \leq C\}$. Let $\left\langle h_{i}\right\rangle_{i \in \omega}$ be an enumeration of $M_{I}$; let $\left\langle\phi_{i}\right\rangle_{i \in \omega}$ be an enumeration of $\Sigma_{2}^{0} \cup \Pi_{2}^{0}$ sentences. Let $P_{0}=Q_{0}=$ id. Let $\left(P_{3 i+1}, Q_{3 i+1}\right)$ be a condition extending $\left(P_{3 i}, Q_{3 i}\right)$ and deciding $\phi_{i}$; let $\left(P_{3 i+2}, Q_{3 i+2}\right)$ be a condition extending $\left(P_{3 i+1}, Q_{3 i+1}\right)$ such that $h_{i} \leq P_{3 i+2}$ and $h_{i} \leq Q_{3 i+2}$. Let $\left(P_{3 i+3}, Q_{3 i+3}\right)$ be a condition extending $\left(P_{3 i+2}, Q_{3 i+2}\right)$ such that for some $n$, either

$$
\begin{gathered}
\left(P_{3 i+3}, Q_{3 i+3}\right) \Vdash “\{\underline{i}\}^{(\underline{B} \oplus C)^{(2)}}(\underline{n}) " \text { diverges, or } \\
\left(P_{3 i+3}, Q_{3 i+3}\right) \Vdash “\{\underline{i}\}^{(\Delta \oplus \underline{B})^{(2)}}(\underline{n})=\underline{m} \text { " for } m \neq f(n) .
\end{gathered}
$$

We must show that such a condition exists.

Otherwise, for every $n$ and every $(P, Q)$ extending $\left(P_{2 i+2}, Q_{3 i+2}\right):(\exists(R, S))$

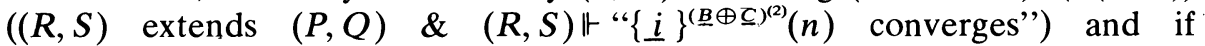
$(P, Q) \Vdash “\{\underline{i}\}^{(B \oplus \underline{C})^{(2)}}(\underline{n})=\underline{k}$ ” then $k=f(n)$. Because $I$ is a case 2 ideal, $M_{I}$

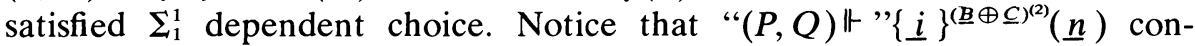
verges" is a $\Sigma_{3}^{0}$ relation between $P \oplus Q, i$ and $n$. $\Sigma_{1}^{1}$ DC. provides an $X \in M_{I}$ such that $(X)_{0}=P_{3 i+2} \oplus Q_{3 i+2}$; and for any $n,\left(R_{n}, S_{n}\right) \Vdash^{\prime \prime}\{\underline{i}\}^{(\underline{B} \oplus \underline{C})^{(2)}}(\underline{n})$ converges"; $\left(R_{n+1}, S_{n+1}\right)$ extends $\left(R_{n}, S_{n}\right)$; where $(X)_{n}=R_{n} \oplus S_{n}$. Note that

$$
\left.\left(R_{n}, S_{n}\right) \& \text { " }\{\underline{i}\}^{(\underline{B} \oplus C)^{(2)}}(\underline{n})=\underline{f(n)}\right) " .
$$

Thus $f \leq X^{(3)}$, contrary to $f \notin M_{I}$. Therefore the desired $\left(P_{3 i+3}, Q_{3 i+3}\right)$ exists.

Stages of the form $3 i+1$ insure that

$$
(B \oplus C)^{(2)}=\left\{m \mid(\exists n)\left(\left(P_{n}, Q_{n}\right) \Vdash \text { “ } \underline{m} \in(\underline{B} \oplus \underline{C})^{(2)}\right),\right) .
$$

Stages of the form $3 i+2$ insure that $M_{I} \subseteq\{f \mid f \leq B \& f \leq C\}$. Stage $3 i+3$ insures that $f \neq\{i\}^{(B \oplus C)^{(2)}}$

ProOf OF THEOREM 5, I UNDER CASE 3. The construction proceeds exactly as under case 2 . However a different argument is required to show that stages of the form $3 i+3$ can be carried out. Suppose not, i.e., suppose that for every $(P, Q)$ extending $\left(P_{3 i+2}, Q_{3 i+2}\right)$ and every $n(\exists(R, S))\left(\left(R_{2} S\right)\right.$ extends $(P, Q)$ \&

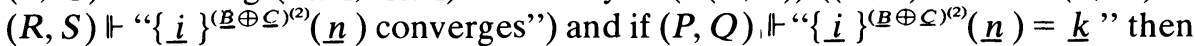
$k=f(n)$. In particular, for any $n,(\exists(R, S))\left((R, S)\right.$ extends $\left(P_{3 i+2}, Q_{3 i+2}\right) \&$

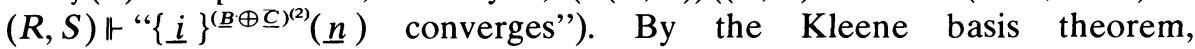
" $(\exists(R, S))$ " may be restricted to " $\left(\exists(R, S) \leq W^{\left(P_{3 i+2} \oplus Q_{3 i+2}\right)}\right)$ ". But $W^{\left(P_{3 i+2} \oplus Q_{3 i+2}\right)} \in$ $M_{I}$. Its third jump computes $f$, implying that $f \in M_{I}$. Contradiction. QED

Thus the best sort of canonicity for nice u.u.b.s, leastness, is available in general only to case 1 ideals. But a weaker sort of canonicity turns out to be available to case 2 ideals: the set of jumps of nice u.u.b.s has a least member. We close with some open questions: Are there, in general, minimal u.u.b.s on ideals? Are there minimal nice u.u.b.s on case 2 and case 3 ideals?

\section{BIBLIOGRAPHY}

[1] R. Boyd, G. Hensel and H. Putnam, A recursion-theoretic characterization of the Ramified Analytical Hierarchy, Transactions, of the American Mathematical Society, vol. 141 (1969), pp. 37-62. 
[2] G. HENSEL and H. PUTNAM, On the notational independence of various hierarchies of degrees of unsolvability, this JOURnAL, vol. 30 (1965), pp. 64-86.

[3] C. JockUSCH and S. Simpson, A degree-theoretic characterization of the Ramified Analytical Hierarchy, Annals of Mathematical Logic, vol. 10 (1976), pp. 1-32.

[4] S. LeEds and H. Putnam, An intrinsic characterization of the hierarchy of the constructible sets of integers, Logic Colloquium '69, North-Holland, Amsterdam.

[5] H. RogERs, The theory of recursive functions and effective computability, McGraw-Hill, New York, 1967.

[6] J. Shoenfield, Mathematical logic, Addison-Wesley, Reading, Massachusetts, 1967.

\author{
DEPARTMENT OF PHILOSOPHY \\ HARVARD UNIVERSITY \\ DEPARTMENT OF PHILOSOPHY \\ CORNELL UNIVERSITY \\ ITHACA, NEW YORK 14850
}

CAMBRIDGE, MASSACHUSETTS 02138 